\title{
Uma Abordagem Física dos Números Perplexos
}

\author{
A physical approach of perplex numbers
}

\author{
Ronni Geraldo Gomes de Amorim*1,2, Wytler Cordeiro dos Santos ${ }^{1,2}$, Lindomar Bonfim Carvalho ${ }^{2}$, \\ Ian Rodrigues Massa ${ }^{2}$ \\ ${ }^{1}$ Universidade de Brasília, Instituto de Física , 70910-900, Brasilia, DF, Brasil \\ ${ }^{2}$ Universidade de Brasilia, Faculdade Gama, Brasilia, DF, Brasil
}

\begin{abstract}
Recebido em 19 de Novembro, 2017. Revisado em 21 de Dezembro, 2017. Aceito em 23 de Dezembro, 2017.
Apresentamos neste trabalho uma revisão pedagógica sobre os números perplexos. O conjunto numérico perplexo, apesar de pouco conhecido, exibe uma álgebra hiperbólica com diversas aplicações na física, por exemplo na Teoria da Relatividade Restrita. Apresentamos a definição de número perplexo, discutimos elementos do cálculo diferencial na variável perplexa e similarmente ao conjunto numérico complexo, definimos as condições de Cauchy-Riemann para a variável perplexa. Aplicamos os resultados para estudar as transformações de Lorentz e a equação de onda no contexto da variável perplexa.
\end{abstract}

Palavras-chave: números perplexos; números hiperbólicos; equação da onda; relatividade restrita.

\begin{abstract}
We present in this work a pedagogical review about the perplex number system. Even though it is lesser well known when compared with its counterpart, we mean the well known Complex number set represented by the letter $\mathbb{C}$, the perplex numbers are related with some hyperbolic algebra with several applications specially in Physics, for instance in the Einstein Special Relativity. For these reasons we start the review presenting the main definitions about the Perplex Numbers, then we discuss some differential calculus based on the perplex algebra. Finally we apply the results for the study of Lorentz Transformations and the wave equation also using the perplex variable.
\end{abstract}

Keywords: perplex numbers; hyperbolic numbers; wave equation; special relativity.

\section{Introdução}

A evolução da física, sobretudo a física teórica, caminha lado a lado com o desenvolvimento da matemática. Quanto mais matemática conhecermos, mais nos tornamos aptos a refletir sobre a natureza e elaborar teorias para explicá-la. Não diferente, o soerguimento do conhecimento a cerca dos conjutos números alavancou o desenvolvimento da física. Se realizarmos uma digressão história sobre a origem dos conjuntos numéricos, perceberemos que os diversos conjuntos surgiram da necessidade humana para solucionar algum problema específico, e a partir do amadurecimento teórico dos conjuntos emergem as aplicações nas ciências. Há evidências que há cinquenta mil anos atrás o homem já sabia contar, e foi dessa noção intuitiva de contagem que surgiram os números naturais 1]. Contudo, havia necessidade de se representar quantias não-inteiras; nesse sentido, há registros de uso de frações no Egito antigo e na Babilônia, enquanto que os números decimais foram introduzidos no continente asiático por volta do século XI 2]. Com as atividades comerciais, surgiu ainda a necessidade de se representar quantias negativas e evidências históricas pontam a utilização de números negativos na China antiga; porém, os primeiros trabalhos com registros da utilização dos números inteiros são do século III, nos quais Diofante procurava soluções para equações algébricas [1,2]. Com os números negativos, as frações e os decimais definia-se os conjuntos dos números inteiros e racionais 11. Na Grécia, o estudo dos pitagóricos a cerca dos segmentos incomensuráveis originou os números irracionais, no entanto há relatos históricos que os babilônios já conheciam algoritmos para o cálculo aproximado de raízes quadradas [2] Apesar de a noção de número imaginário ter surgido no século XVI, a sistematização do conjunto dos números complexos só foi realizada no século XIX com a sua representação no plano complexo [1,2]. Tanto é verdade que Isaac Newton dividia os números em três tipos: inteiros, frações e irracionais. Contudo, nos séculos XIX e XX ocorreu a axiomatização da teoria de conjuntos [1], e a partir de então a aplicação dos números na física tornouse mais eminente. Por exemplo, podemos citar o uso dos números complexos ao estudo de circuitos elétricos e fasores. Ainda no século XIX, Hamilton introduziu os quaternions (uma extensão dos números complexos), a partir dos quais pode-se mostrar a origem dos produtos escalar e vetorial, com muitas aplicações na física [3 5$]$. Neste trabalho, apresentaremos um conjunto numérico

*Endereço de correspondência: ronniamorim@gmail.com 
denominado números perplexos ou hiperbólicos, e mostraremos algumas aplicações desses números na física.

Os números perplexos, também denominados números hiperbólicos, foram apresentados pela primeira vez em 1919, no trabalho de L. E. Dickson 6]. Contudo, o interesse no estudo desses números foi revigorado a partir de 1981, quando Paul Fjelstad, auxiliado por quatro estudantes, utilizou os números perplexos para explicar o fenômeno supraluminal [7]. O intuito de Fjelstad era propor aos seus estudantes o desafio da criar algo novo em matemática. Para sua surpresa, os estudantes investigaram a relação entre a trigonometria hiperbólica e a relatividade espacial, desenvolvendo assim importantes aspectos da álgebra dos números perplexos, além de aplicá-la em contextos físicos 77. A motivação essencial na proposição desse novo conjunto de números emergiu da analogia entre a trigonometria circular e a hiperbólica. Em particular, a identidade fundamental da trigonometria hiperbólica, $\cosh ^{2} x-\sinh ^{2} x=1$, difere da relação análoga na trigonometria circular, $\cos ^{2} x+\sin ^{2} x=1$, somente por um sinal. Além disso, sabemos que a definição da unidade imaginária como $i^{2}=-1$, induz à relação de Euler, $e^{i \theta}=\cos \theta+i \sin \theta$. A partir daí surge a indagação: dada a analogia entre as funções trigonométricas hiperbólicas e as circulares, seria possível estabelecer a definição de uma unidade análoga à unidade imaginária dos complexos a fim de se obter uma relação similar à de Euler que seja válida para as funções trigonométricas hiperbólicas? A resposta é positiva, e isso é realizado mediante a introdução da unidade perplexa. Neste trabalho, vamos representar a unidade perplexa por $p$, a qual satisfaz a condição $p^{2}=1[8]$. Ademais, o conjunto dos números perplexos será representado pelo símbolo $\mathbb{P}$. O objetivo deste artigo é trazer uma revisão da álgebra dos números perplexos, apresentando as suas principais aplicações na Física.

A apresentação deste trabalho será constituída dos seguintes tópicos. Na seção 2, apresentamos a definição dos números perplexos e suas principais propriedades. Na seção 3, discutimos o conceito de norma e construímos a forma polar de uma número perplexo. Na seção 4, é apresentada a teoria sobre funções analíticas na variável perplexa. Na seção 5, as aplicações na Física são discutidas. Por fim, apresentamos as considerações finais.

\section{Definição e Operações}

Em analogia com os números complexos, um número perplexo pode ser representado pela forma algébrica

$$
w=x+y p,
$$

onde $x, y \in \mathbb{R}$, ou seja, representam respectivamente, a parte real de $w$ e a parte perplexa de $w$, que pode ser escrito como: $x=\operatorname{Re}\{w\}$ e $y=\operatorname{Per}\{w\}$.

Portanto, dois números perplexos $w_{1}=x_{1}+y_{1} p$ e $w_{2}=x_{2}+y_{2} p$ são iguais, se e somente se, $x_{1}=x_{2}$ e $y_{1}=y_{2}$.
Com esta representação, as operações de soma e subtração são definidas de forma análoga à dos números complexos, ou seja, dados $w_{1}$ e $w_{2}$ definidos por: $w_{1}=x_{1}+y_{1} p$ e $w_{2}=x_{2}+y_{2} p$, tem-se

$$
\begin{aligned}
& w_{1}+w_{2}=\left(x_{1}+x_{2}\right)+\left(y_{1}+y_{2}\right) p \\
& w_{1}-w_{2}=\left(x_{1}-x_{2}\right)+\left(y_{1}-y_{2}\right) p .
\end{aligned}
$$

A multiplicação pode ser realizada utilizando-se a propriedade distributiva e a definição $p^{2}=1$. É importante que o leitor entenda que $p$ não pertence ao conjunto dos números reais, o que exclui a possibilidade de $p=1$ ou $p=-1$. Com esta definição, a tabela de multiplicação envolvendo a unidade perplexa fica estabelecida com as relações: $p p=p^{2}=1, p 1=p, 1 p=p, 11=1$. Assim, temos

$$
\begin{aligned}
w_{1} w_{2} & =\left(x_{1}+y_{1} p\right)\left(x_{2}+y_{2} p\right) \\
& =x_{1} x_{2}+x_{1} y_{2} p+y_{1} x_{2} p+y_{1} y_{2} p^{2} \\
& =\left(x_{1} x_{2}+y_{1} y_{2}\right)+\left(x_{1} y_{2}+x_{2} y_{1}\right) p .
\end{aligned}
$$

Com isso teremos então

$$
\begin{aligned}
\operatorname{Re}\left\{w_{1} w_{2}\right\} & =x_{1} x_{2}+y_{1} y_{2} \\
\operatorname{Per}\left\{w_{1} w_{2}\right\} & =x_{1} y_{2}+x_{2} y_{1} .
\end{aligned}
$$

Ademais adotando a notação de par ordenado para o número perplexo $w=(x, y)$, com isto teremos $w_{1} w_{2}=\left(x_{1}, y_{1}\right)\left(x_{2}, y_{2}\right)=\left(x_{1} x_{2}+y_{1} y_{2}, x_{1} y_{2}+x_{2} y_{1}\right)$.

Desse modo podemos definir o conjugado perplexo de um número $w=x+y p$ como sendo $\bar{w}=x-y p$.

Outra relação importante obtém-se ao aplicar a definição de multiplicação entre $w$ e seu perplexo conjugado:

$$
w \bar{w}=x^{2}-y^{2} .
$$

Note que $w \bar{w}=0$ se e somente se $|x|=|y|$. Portanto, o conjunto dos perplexos não forma uma algébra divisória, em outras palavras, podemos ter dois números perplexos não nulos tal que $w_{1} w_{2}=0$. Esta é uma característica que pode ser encontrada no cálculo com matrizes. Por isso os números perplexos possuem mais propriedades próximas às das matrizes do que dos números complexos (que formam uma algébra divisória). Como veremos adiante, tantos as matrizes quanto os perplexos guardam estreita relação com o cone de luz da relatividade especial.

Neste ponto, estamos aptos a definir a divisão entre dois números perplexos. Para isso, vamos imaginar a divisão como sendo a operação inversa da multiplicação, ou seja, se $w_{3}=\frac{w_{1}}{w_{2}}$, temos $w_{1}=w_{2} w_{3}$. O leitor deve se atentar que a divisão só é definida se $w_{2} \bar{w}_{2}$ for real e diferente de zero. Sendo assim, se $w_{1}=x_{1}+y_{1} p$, $w_{2}=x_{2}+y_{2} p$ e $w_{3}=x_{3}+y_{3} p$, temos

$$
w_{1}=w_{2} w_{3} .
$$

Como $w_{2} \bar{w}_{2} \neq 0$, podemos multiplicar ambos os lados da última expressão por $\bar{w}_{2}$, obtendo

$$
\bar{w}_{2} w_{1}=\bar{w}_{2} w_{2} w_{3} .
$$


Como $w_{2} \bar{w}_{2}$ é real, tem-se

$$
w_{3}=\frac{\bar{w}_{2} w_{1}}{\bar{w}_{2} w_{2}} .
$$

E finalmente encontramos que

$$
w_{3}=\frac{\left(x_{1} x_{2}-y_{1} y_{2}\right)+\left(y_{1} x_{2}-x_{1} y_{2}\right) p}{x_{2}^{2}-y_{2}^{2}},
$$

desde que $\left|x_{2}\right| \neq\left|y_{2}\right|$.

Considerando $w+\bar{w}=2 x$ e $w-\bar{w}=2 y p$, podemos escrever

$$
\begin{aligned}
\operatorname{Re}\{w\} & =\frac{w+\bar{w}}{2} \\
\operatorname{Per}\{w\} & =\frac{p(w-\bar{w})}{2} .
\end{aligned}
$$

É relevante visualizarmos as potencias da unidade perplexa, ou seja, $p^{0}=1, p^{ \pm 1}=p, p^{ \pm 2}=1, p^{ \pm 3}=p$, $p^{ \pm 4}=1$, e assim sucessivamente. Ou seja

$$
p^{k}=\left\{\begin{array}{l}
1, \text { se } \mathrm{k} \text { é par } \\
p, \text { se k é impar. }
\end{array}\right.
$$

Tendo definido as operações básicas do conjunto dos números perplexos, na próxima seção discorreremos sobre as propriedades inerentes à definição de norma.

\section{Norma e Forma Hiperbólica de um Número Perplexo}

No contexto dos números perplexos, definimos uma aplicação de um número $w=x+y p$, representada por $\lambda(w)$ como

$$
\lambda(w)=w \bar{w}=x^{2}-y^{2} .
$$

Como $x$ e $y$ são reais, $\lambda(w) \in \mathbb{R}$, podemos utilizar o conceito convencional de módulo para essa aplicação. E ainda utilizaremos da função sinal para propriamente definir norma perplexa, ou seja,

$$
\operatorname{sgn}(x)=\left\{\begin{array}{rll}
1 & \text { se } & x>0 \\
0 & \text { se } & x=0 \\
-1 & \text { se } & x<0
\end{array}\right.
$$

Por fim, a norma de um número perplexo $w$ é dada por

$$
\|w\|=\operatorname{sgn}(\lambda(w)) \sqrt{|\lambda(w)|}
$$

Note que a norma dada na Eq. (7) não é positivo-definida. Essa característica é fundamental para estabelecermos a relação entre os números perplexos e o espaço de Minkowsky.

Para estabelecermos a forma hiperbólica de um número perplexo, antes façamos um estudo da exponencial $e^{p \theta}$. Fazendo a expansão de Maclaurin de $e^{x}$ e substituindo $x$ por $p \theta$ obteremos:

$$
e^{p \theta}=1+p \theta+\frac{1}{2 !}(p \theta)^{2}+\frac{1}{3 !}(p \theta)^{3}+\cdots
$$

$$
e^{p \theta}=\left(1+\frac{1}{2 !} \theta^{2}+\frac{1}{4 !} \theta^{4}+\cdots\right)+p\left(\theta+\frac{1}{3 !} \theta^{3}+\cdots\right) .
$$

Lembrando as expressões de $\cosh \theta=1+\frac{1}{2 !} \theta^{2}+\frac{1}{4 !} \theta^{4}+\cdots$ e $\sinh \theta=\theta+\frac{1}{3 !} \theta^{3}+\cdots$, temos que

$$
e^{p \theta}=\cosh \theta+p \sinh \theta .
$$

De forma bastante similar $e^{-p \theta}=\cosh \theta-p \sinh \theta$.

Agora, nosso objetivo é representar $w=x+y p$ na forma hiperbólica. É importante mencionar que a forma cartesiana de um número perplexo, $w=x+y p$, depende da relação de ordem entre $x$ e $y$. Tomemos então $w \bar{w}=$ $\lambda(w)$, assim, temos

$$
w \bar{w}=\lambda(w)=x^{2}-y^{2} .
$$

Dividindo a Eq. 8 por $\lambda$, supondo $\lambda(w) \neq 0$, obtemos

$$
\frac{x^{2}}{\lambda}-\frac{y^{2}}{\lambda}=1 \text {, }
$$

ou seja, $w$ está numa hiperbole equilátera. Neste caso, podemos tomar $x=\lambda \cosh \theta$ e $y=\lambda \sinh \theta$, uma vez que $\cosh ^{2} \theta-\sinh ^{2} \theta=1$. Fazendo isso, obtemos

$$
\tanh \theta=\frac{y}{x}
$$

$$
\theta=\ln \left(\sqrt{\frac{x+y}{x-y}}\right) .
$$

Com isso, a forma hiperbólica de $w$ nos diferentes casos será dada por:

- Se $|x|>|y|$, existem dois casos:

- Com $x>|y|$, escrevemos $w=\|w\|(\cosh \theta+p \sinh \theta)=\sqrt{\lambda}(\cosh \theta+$ $p \sinh \theta)=\|w\| e^{p \theta}$

- Com $x<-|y|$, basta usar $w=-1(-w)$, onde $-w$ já possuí forma hiperbólica bem definida.

- Já se $|x|<|y|$,temos:

- Para $y>|x|, w=p(p w)=p\|p w\| e^{p \theta}$. Note que $\theta \in\left[-\frac{\pi}{2}, \frac{\pi}{2}\right]$, pois representa o ângulo de pw.

- Para $y<-|x|, w=-p(-p w)=-p||-$ $p w \| e^{p \theta}$. Novamente, $\theta \in\left[-\frac{\pi}{2}, \frac{\pi}{2}\right]$, pois representa o ângulo de $-p w$.

Com a definição de norma já estabelecida, é natural a definição do produto interno, $\left\langle w_{1}, w_{2}\right\rangle$, entre dois números perplexos $w_{1}=x_{1}+p y_{1}$ e $w_{2}=x_{2}+p y_{2}$ como

$$
\begin{gathered}
\left\langle w_{1}, w_{2}\right\rangle=w_{1} \bar{w}_{2}, \\
\left\langle w_{1}, w_{2}\right\rangle=x_{1} x_{2}-y_{1} y_{2},
\end{gathered}
$$

em que $\bar{w}_{2}=x_{2}-p y_{2}$.

Nesse caminho, a desiguladade de Cauchy-Schwarz perplexa é dada por

$$
\left\langle w_{1}, w_{2}\right\rangle^{2} \geq \lambda\left(w_{1}\right) \lambda\left(w_{2}\right)
$$


Prova :

$$
\begin{aligned}
& {\left[\left(x_{1} y_{2}\right)-\left(x_{2} y_{1}\right)\right]^{2} \geq 0} \\
& \left(x_{1} y_{2}\right)^{2}+\left(x_{2} y_{1}\right)^{2} \geq 2\left(x_{1} y_{2}\right)\left(x_{2} y_{1}\right) \\
& -\left[\left(x_{1} y_{2}\right)^{2}+\left(x_{2} y_{1}\right)^{2}\right] \leq-2\left(x_{1} y_{2}\right)\left(x_{2} y_{1}\right) \\
& \left(x_{1} x_{2}\right)^{2}+\left(y_{1} y_{2}\right)^{2}-\left[\left(x_{1} y_{2}\right)^{2}+\left(x_{2} y_{1}\right)^{2}\right]=\lambda\left(w_{1}\right) \lambda\left(w_{2}\right) \\
& \left(x_{1} x_{2}\right)^{2}+\left(y_{1} y_{2}\right)^{2}-2\left(x_{1} y_{2}\right)\left(x_{2} y_{1}\right)=\left\langle w_{1}, w_{2}\right\rangle^{2} \therefore \\
& \left\langle w_{1}, w_{2}\right\rangle^{2} \geq \lambda\left(w_{1}\right) \lambda\left(w_{2}\right) .
\end{aligned}
$$

Potências e raízes de números perplexos podem ser facilmente obtidas se utilizarmos a forma polar ou exponencial. Fica a cargo do leitor encontrar expressões análogas às fórmulas de Moivre dos complexos.

\section{Funções Analíticas no Plano Perplexo}

Nesta seção, discutiremos um pouco sobre as funções analíticas no plano perplexo, obtendo condições análogas às de Cauchy-Riemann 9]. Para esse fim, definamos função na variável perplexa.

Consideramos como função de uma variável perplexa $f(w)$, em que $w=x+p y$, como fuma função $f: \mathbb{P} \rightarrow \mathbb{P}$, ou seja, uma função que leva números perplexos a números perplexos. Nesse sentido, qualquer função $f(w)$ pode ser escrita na forma:

$$
f(w)=u(x, y)+p v(x, y)
$$

onde $u(x, y)$ e $v(x, y)$ são funções das variáveis reais $x$ e $y$. E ainda, $u(x, y)=\operatorname{Re}\{f(w)\}$ e $v(x, y)=\operatorname{Per}\{f(w)\}$.

Como exemplo, tomemos a função $f(w)=w^{2}$, essa função pode ser escrita como

$f(w)=(x+p y)^{2}=x^{2}+2 p x y+y^{2}=\left(x^{2}+y^{2}\right)+p(2 x y)$. Neste caso, identificamos $u(x, y)=\left(x^{2}+y^{2}\right)$ e $v(x, y)=$ $2 x y$.

A derivada no plano perplexo é então definida de forma similar à derivada no plano complexo, ou seja, se $f(w)$ é uma função de variável perplexa e $w$ pertence ao seu domínio, então definimos a derivada de $f(w)$ em relação a $w$ como

$$
f^{\prime}(w)=\frac{d f(w)}{d w}=\lim _{\Delta w \rightarrow 0} \frac{f(w+\Delta w)-f(w)}{\Delta w} .
$$

Se o limite dado na Eq. 10 existir, dizemos que $f(w)$ é diferenciável. Assim, se $f(w)$ é diferenciável, $\Delta w$ deve se aproximar de zero por caminhos diferentes e ter o mesmo limite por todos eles. Os únicos caminhos que não podem ser percorridos, são caminhos que incluem as retas $y=x$ e $y=-x$, pois $f(w)$ não é inversível sobre elas.

Assim, temos:

$$
\begin{array}{r}
f^{\prime}\left(w_{0}\right)=\lim _{\substack{\Delta x \rightarrow 0 \\
\Delta y \rightarrow 0}}\left(\frac{u\left(x_{0}+\Delta x, y_{0}+\Delta y\right)-u\left(x_{0}, y_{0}\right)}{\Delta x+p \Delta y}\right. \\
\left.+p \frac{v\left(x_{0}+\Delta x, y_{0}+\Delta y\right)-v\left(x_{0}, y_{0}\right)}{\Delta x+p \Delta y}\right) .
\end{array}
$$

Como esse limite é igual por qualquer caminho do plano perplexo, escolhamos dois caminhos particulares, quais sejam $\Delta x \rightarrow 0$ e $\Delta y \rightarrow 0$, respectivamente. Para o primeiro caminho, $\Delta x \rightarrow 0$, temos

$$
\begin{aligned}
f^{\prime}\left(w_{0}\right) & =\lim _{\Delta y \rightarrow 0}\left(\frac{u\left(x_{0}, y_{0}+\Delta y\right)-u\left(x_{0}, y_{0}\right)}{p \Delta y}\right. \\
& \left.+p \lim _{\Delta y \rightarrow 0} \frac{v\left(x_{0}, y_{0}+\Delta y\right)-v\left(x_{0}, y_{0}\right)}{p \Delta y}\right) \\
& =p \frac{\partial u}{\partial y}+\frac{\partial v}{\partial y} .
\end{aligned}
$$

Para o segundo caminho, $\Delta y=0$, obtemos

$$
\begin{aligned}
f^{\prime}\left(w_{0}\right) & =\lim _{\Delta x \rightarrow 0}\left(\frac{u\left(x_{0}+\Delta x, y_{0}\right)-u\left(x_{0}, y_{0}\right)}{\Delta x}\right. \\
& \left.+p \lim _{\Delta x \rightarrow 0} \frac{v\left(x_{0}+\Delta x, y_{0}\right)-v\left(x_{0}, y_{0}\right)}{\Delta y}\right) \\
& =\frac{\partial u}{\partial x}+p \frac{\partial v}{\partial x} .
\end{aligned}
$$

Como os limites devem ser iguais, concluímos que

$$
p \frac{\partial u}{\partial y}+\frac{\partial v}{\partial y}=\frac{\partial u}{\partial x}+p \frac{\partial v}{\partial x} .
$$

O que nos leva a

$$
\begin{aligned}
& \frac{\partial u}{\partial x}=\frac{\partial v}{\partial y} \\
& \frac{\partial u}{\partial y}=\frac{\partial v}{\partial x}
\end{aligned}
$$

as quais são as condições de Cauchy-Riemann na variável perplexa.

A partir das condições de Cauchy-Riemann, podemos deduzir relações importantes, as quais serão utilizadas nas aplicações físicas do cálculo perplexo. Para isso, vamos derivar a primeira equação das condições de CauchyRiemann em relação a $x$ e a segunda em relação a $y$, obtendo, respectivamente,

$$
\begin{aligned}
\frac{\partial^{2} u}{\partial x^{2}} & =\frac{\partial^{2} v}{\partial y \partial x} \\
\frac{\partial^{2} u}{\partial y^{2}} & =\frac{\partial^{2} v}{\partial x \partial y} .
\end{aligned}
$$

Como $\frac{\partial^{2} v}{\partial y \partial x}=\frac{\partial^{2} v}{\partial x \partial y}$, por conta da diferenciabilidade, obtemos

$$
\frac{\partial^{2} u}{\partial x^{2}}-\frac{\partial^{2} u}{\partial y^{2}}=0,
$$

ou seja, se $f(w)$ é diferenciável, as funções $u$ e $v$ satisfazem a equação de D'Alembert 10,11. É interessante compararmos este último resultado ao panorama da variável 
complexa. Enquanto no âmbito dos números complexos as condições de Cauchy-Riemann induzem a equação de Laplace, no caso dos números perplexos as condições de Cauchy-Riemann induzem a equação de D'Alembert. Este fato implicará em instigantes aplicações na física.

Na próxima seção, apresentaremos as consequências físicas do cálculo na variável perplexa.

\section{Aplicações na Física}

\subsection{Equação de Onda $(1+1)$}

Como primeira aplicação na Física, apresentamos a equação de onda unidimensional. Essa equação é bem conhecida da literatura e tem a forma,

$$
\frac{\partial^{2} \psi(x, t)}{\partial x^{2}}-\frac{1}{v^{2}} \frac{\partial^{2} \psi(x, t)}{\partial t^{2}}=0
$$

a qual descreve uma onda que oscila na direção $x$ com o passar do tempo $t$. A função $\psi(x, t)$ satisfaz a equação de D'Alembert 10,11], isso sugere que possamos encontrar a solução para a equação de onda usando a variável perplexa. Escolhendo $w=x+p v t$ e $\bar{w}=x-p v t$, as derivadas na Eq.13 podem se escritas como

$$
\begin{gathered}
\frac{\partial}{\partial x}=\frac{\partial}{\partial w}+\frac{\partial}{\partial \bar{w}} \\
\frac{\partial}{\partial t}=p v \frac{\partial}{\partial w}-p v \frac{\partial}{\partial \bar{w}} \\
\frac{\partial^{2}}{\partial x^{2}}=\frac{\partial^{2}}{\partial w^{2}}+2 \frac{\partial^{2}}{\partial w \bar{w}}+\frac{\partial^{2}}{\partial \bar{w}^{2}}, \\
\frac{\partial^{2}}{\partial t^{2}}=v^{2} \frac{\partial^{2}}{\partial w^{2}}-2 v^{2} \frac{\partial^{2}}{\partial w \bar{w}}+v^{2} \frac{\partial^{2}}{\partial \bar{w}^{2}} .
\end{gathered}
$$

Com isso, a Eq.13 torna-se

$$
\frac{\partial^{2} \psi(x, t)}{\partial x^{2}}-\frac{1}{v^{2}} \frac{\partial^{2} \psi(x, t)}{\partial t^{2}}=4 \frac{\partial^{2} \psi(w, \bar{w})}{\partial w \partial \bar{w}} .
$$

E a equação que nos resta resolver é a seguinte

$$
\frac{\partial^{2} \psi(w, \bar{w})}{\partial w \partial \bar{w}}=0
$$

cuja solução é facilmente encontrada por integração direta, obtendo-se

$$
\psi(w, \bar{w})=g_{1}(w)+g_{2}(\bar{w})
$$

ou seja,

$$
\psi(x, t)=g_{1}(x+v t)+g_{2}(x-v t) .
$$

A utilização da variável perplexa nos permite ainda resolver alguns casos das equações de onda não-homogêneas de forma muito fácil. Considere por exemplo a equação

$$
\frac{\partial^{2} \psi(x, t)}{\partial x^{2}}-\frac{1}{v^{2}} \frac{\partial^{2} \psi(x, t)}{\partial t^{2}}=\frac{K}{x^{2}-(v t)^{2}},
$$

essa equação pode ser escrita como

$$
\frac{\partial^{2} \psi(w, \bar{w})}{\partial w \partial \bar{w}}=\frac{K}{w \bar{w}}
$$

com isso, podemos obter uma solução particular $\psi_{p}(w, \bar{w})$ considerando nulas demais constantes de integração e definindo uma função logaritmo ( para $x>|y|$ ), inversa da exponencial $e^{p \theta}$, sendo dada por

$$
\log (w) \doteq \ln \sqrt{|\lambda(w)|}+p \ln \left(\sqrt{\frac{x+y}{x-y}}\right) .
$$

E assim, temos como solução

$$
\begin{aligned}
\psi_{p}(w, \bar{w}) & =\frac{K}{4} \log (w) \log (\bar{w})=\frac{K}{4} \log (w) \overline{\log (w)} \\
& =\frac{K}{4}\|\log (w)\|^{2}
\end{aligned}
$$

Note que $\psi_{p} \in \mathbb{R}$, como esperado.

A solução em função de variáveis reais, $x$ e $v t$, é dada por

$$
\psi_{p}(x, v t)=\frac{K}{4}\left[\ln ^{2} \sqrt{x^{2}-(v t)^{2}}-\ln ^{2}\left(\sqrt{\frac{x+v t}{x-v t}}\right)\right] .
$$

Outras soluções podem ser obtidas considerando demais regiões do plano perplexo, basta uma definição apropriada da função logaritmo. Fica como tarefa para o leitor analisar as soluções em outras regiões do plano perplexo.

\subsection{Transformações de Lorentz $(1+1)$}

Sejam as transformações de Lorentz em uma direção denominada comumente de boost na direção do eixo $x$ 11, 12 ,

$$
\left\{\begin{array}{l}
x^{\prime}=\gamma(x-v t) \\
c t^{\prime}=\gamma\left(c t-\frac{v}{c} x\right),
\end{array}\right.
$$

onde as coordenadas $(x, t)$ são de um sistema de referências inercial $\mathcal{O}$ e $\left(x^{\prime}, t^{\prime}\right)$ são coordenadas em um sistema de referências inercial $\mathcal{O}^{\prime}$. O fator de Lorentz é

$$
\gamma=\frac{1}{\sqrt{1-\frac{v^{2}}{c^{2}}}}
$$

e $c$ é a velocidade da luz. Essas transformações podem ser denotadas como uma transformação linear na forma matricial,

$$
\left(\begin{array}{c}
x^{\prime} \\
c t^{\prime}
\end{array}\right)=\left(\begin{array}{cc}
\gamma & -\frac{v}{c} \gamma \\
-\frac{v}{c} \gamma & \gamma
\end{array}\right)\left(\begin{array}{l}
x \\
c t
\end{array}\right)
$$

Isso nos leva ao invariante de Lorentz:

$$
x^{2}-c^{2} t^{\prime 2}=x^{2}-c^{2} t^{2} .
$$

O determinante da matrix de transformação é:

$$
\operatorname{det}\left(\begin{array}{cc}
\gamma & -\frac{v}{c} \gamma \\
-\frac{v}{c} \gamma & \gamma
\end{array}\right)=\gamma^{2}-\frac{v^{2}}{c^{2}} \gamma^{2}=\gamma^{2}\left(1-\frac{v^{2}}{c^{2}}\right)=1 \text {. }
$$


As transformações de Lorentz então são transformações ortogonais onde podemos fazer a seguinte mudança de variável,

$$
\gamma=\cosh \theta \quad \text { e } \quad \frac{v}{c} \gamma=\sinh \theta,
$$

tal que

$$
\tanh \theta=\frac{v}{c} .
$$

Dessa forma as transformações de Lorentz se tornam uma "rotação" do sistema de coordenadas $(x, t)$ dada por 13 :

$$
\left(\begin{array}{l}
x^{\prime} \\
c t^{\prime}
\end{array}\right)=\left(\begin{array}{cc}
\cosh \theta & -\sinh \theta \\
-\sinh \theta & \cosh \theta
\end{array}\right)\left(\begin{array}{l}
x \\
c t
\end{array}\right) .
$$

Se denotarmos o vetor da Relatividade Especial $(x, t)$ no sistema de coordenadas inercial $\mathcal{O}$ por um número perplexo,

$$
w=x+p c t
$$

então obtemos o invariante de Lorentz 20,

$$
w \bar{w}=x^{2}-c^{2} t^{2} .
$$

Assim como os módulos dos vetores na Relatividade Especial [12], da mesma forma teremos que: (i) se $w \bar{w}<0$ o número perplexo pode ser denominado de tipo tempo, (ii) se $w \bar{w}>0$ o número perplexo pode ser denominado de tipo espaço e (iii) se $w \bar{w}=0$ o número perplexo será denominado do tipo luz. O vetor $(x, t)$ da Relatividade Especial no sistema de coordenadas inercial $\mathcal{O}^{\prime}$ será o número perplexo:

$$
w^{\prime}=x^{\prime}+p c t^{\prime}
$$

onde podemos usar as transformações de Lorentz 24 e obter

$$
\begin{aligned}
w^{\prime} & =x \cosh \theta-c t \sinh \theta+p(c t \cosh \theta-x \sinh \theta) \\
& =x(\cosh \theta-p \sinh \theta)+p c t(\cosh \theta-p \sinh \theta) \\
& =x e^{-p \theta}+p c t e^{-p \theta} \\
& =(x+p c t) e^{-p \theta}
\end{aligned}
$$

então na liguagem dos números perplexos uma transformação de Lorentz será dada por:

$$
w^{\prime}=w e^{-p \theta} .
$$

Se tivermos uma partícula se movendo em relação aos dois sistemas de referências inerciais $\mathcal{O}$ e $\mathcal{O}^{\prime}$ com velocidade constante $u^{\prime}$, então a transformação de Lorentz entre o referencial da partícula $\mathcal{O}^{\prime \prime}$ e o referencial $\mathcal{O}^{\prime}$ é dado por:

$$
w^{\prime \prime}=w^{\prime} e^{-p \phi} \text {. }
$$

Então um observador no sistema de referências $\mathcal{O}$ observa a partícula através das transformações,

$$
w^{\prime \prime}=w^{\prime} e^{-p \phi}=w e^{-p \theta} e^{-p \phi}=w e^{-p(\theta+\phi)} .
$$

Da expressão para a velocidade dada na equação $\sqrt{23}$, nós temos que a velocidade da partícula vista pelo sistema de referências $\mathcal{O}^{\prime}$ é $u^{\prime}$ dado por:

$$
\tanh \phi=\frac{u^{\prime}}{c} \text {. }
$$

Da transformação de Lorentz em termos de números perplexos na equação (31), a velocidade observada no referencial inercial $\mathcal{O}$ deve ser $u$

$$
\tanh (\theta+\phi)=\frac{u}{c} \text {. }
$$

Das identidades das funções hiperbólicas, sabemos que:

$$
\tanh (\theta+\phi)=\frac{\tanh (\theta)+\tanh (\phi)}{1+\tanh (\theta) \tanh (\phi)},
$$

de forma que

$$
\frac{u}{c}=\frac{\frac{v}{c}+\frac{u^{\prime}}{c}}{1+\frac{v}{c} \frac{u^{\prime}}{c}},
$$

ou seja,

$$
u=\frac{v+u^{\prime}}{1+\frac{u^{\prime} v}{c^{2}}}
$$

em acordo com as transformações de velocidades na Relatividade Especial [11.

A energia relativística de uma partícula livre é dada por:

$$
E^{2}-\|\boldsymbol{P}\|^{2} c^{2}=m^{2} c^{4},
$$

onde $E=\gamma m c^{2}$ é a energia e $\|\boldsymbol{P}\|=\gamma m v$ é o momentum da partícula [11,12], sendo $m$ a massa de repouso.

Também é possível expressar a energia/momentum relativístico de uma partícula por um número perplexo,

$$
w=E+p\|\boldsymbol{P}\| c
$$

de forma que o módulo será dado por:

$$
w \bar{w}=E^{2}-\|\boldsymbol{P}\|^{2} c^{2}
$$

ou então

$$
w \bar{w}=m^{2} c^{4} .
$$

Um número perplexo na forma polar, dado por,

$$
w=\|w\| e^{p \theta}
$$

resulta que para a quantidade perplexa para energia da partícula será:

$$
w=m^{2} c^{4}(\cosh \theta+p \sinh \theta),
$$

comparando a expressão acima com a equação (37), obtemos que:

$$
\cosh \theta=\frac{E}{m c^{2}} \quad \text { e } \quad \sinh \theta=\frac{\|\boldsymbol{P}\| c}{m c^{2}} .
$$

Com essas condições pode-se verificar que a tangente hiperbólica será

$$
\tanh \theta=\frac{\|\boldsymbol{P}\| c}{E}=\frac{\gamma m v c}{\gamma m c^{2}}=\frac{v}{c},
$$


que é a mesma expressão para a velocidade da equação (23).

A natureza quântica da luz nos diz que a energia e o módulo do momentum do fóton são respectivamente [11],

$$
E=h f=\frac{h c}{\lambda} \quad \text { e } \quad\|\boldsymbol{P}\|=\frac{h}{\lambda},
$$

onde $h$ é a constante de Planck e $\lambda$ é o comprimento de onda do fóton. A quantidade perplexa para a energia (37) para um fóton de comprimento de onda $\lambda$ resulta em:

$$
w=\frac{h c}{\lambda}(1+p) \text {. }
$$

No caso de fótons que são partículas não massivas, $m=0$, terá módulo nulo de acordo com a expressão 38 ,

$$
w \bar{w}=0 .
$$

Então a expressão para a velocidade do fóton será dado pela expressão 42 que resultará em:

$$
\tanh \theta=\frac{\|\boldsymbol{P}\| c}{E}=\frac{\frac{h}{\lambda} c}{\frac{h c}{\lambda}}=1 .
$$

Da equação 23 temos para o fóton, $v=c$.

Como o módulo $\|w\|$ do número perplexo da energia dos fótons são nulos $(w \bar{w}=0)$ e pela análise de que a tangente hiperbólica assume valor igual a um somente no limite em que $\theta \rightarrow \infty$, veja o gráfico da Figura 1 , pode-se afirmar que não é possível expressar o número perplexo da energia do fóton na forma polar.

\section{Considerações Finais}

Neste trabalho apresentamos de forma pedagógica uma revisão sobre números perplexos. Discutimos desde a definição do conjunto dos números perplexos até a teoria de funções analíticas na variável perplexa. Também

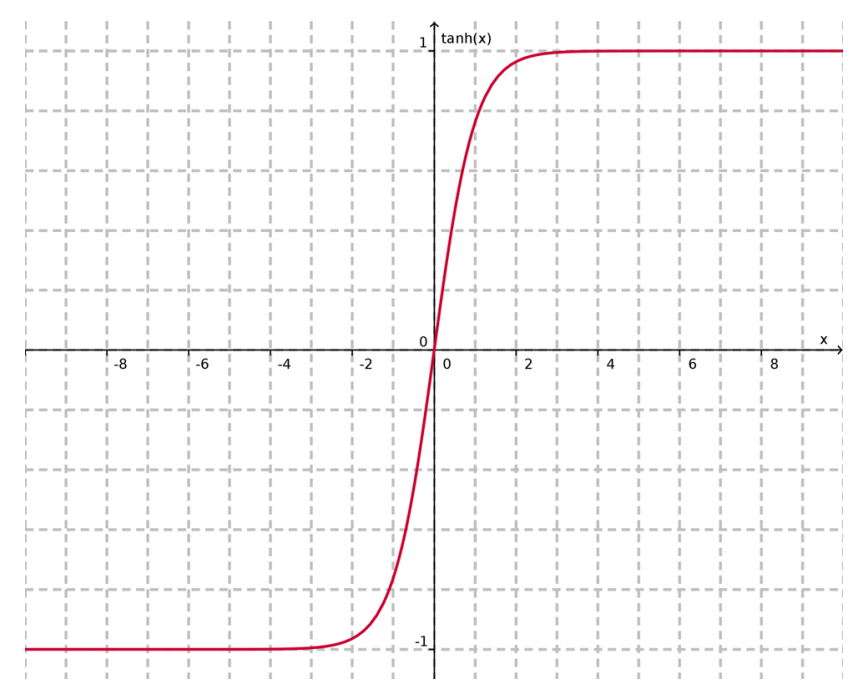

Figura 1: Tangente hiperbólica apresentamos as condições de Cauchy-Riemman válidas no cálculo de uma variável perplexa. A diferença se dá no sinal de uma das condições, ou melhor, enquanto na varíavel complexa uma das condições de CauchyRiemman possui um sinal negativo [9], nos números perplexos ambas são positivas (11). A consequência deste fato é que nos números perplexos obtém-se a equação de D'Alembert, enquanto na variável complexa é obtida a equação de Laplace. Com tais resultados, discutimos algumas aplicações na física, tais como a equação de onda e as transformações de Lorentz. Como perspectiva, esperamos aprofundar no desenvolvimento do cálculo da variável perplexa, estudando o teorema de Cauchy e o teorema do Resíduo nesse arcabouço teórico com possíveis aplicações na física.

\section{Agradecimentos}

Os autores agradecem ao $\mathrm{CNPq}$ pelo auxílio financeiro.

\section{Referências}

[1] H. Eves, Introdução à História da Matemática (Editora Unicamp, Campinas, 2004), p. 77.

[2] C.B. Boyer, História da Matemática (Edgard Blucher, São Paulo, 1974), p. 36.

[3] P.G. Tait, An Elementary Treatise on Quaternions (Cambridge University Press, Cambridge, 1890), p. 122.

[4] S. Adler, Quaternionic Quantum Mechanics and Quantum Fields (Oxford University Press, New York, 1995), p. 201.

[5] M.J. Menon, Rev. Bras. Ens. Fís. 31, 2305 (2009).

[6] L.E. Dickson, Ann. Math. Series 2 20, 155 (1919).

[7] P. Fjelstad, American Journal of Physics 54, 416 (1986).

[8] A.K.T. Assis, International Journal of Mathematical Education in Science and Technology 22, 555 (1991).

[9] J.W. Brown and R.V. Churchill, Variáveis Complexas e Aplicações (McGraw-Hill, New York, 2015), p. 70.

[10] R.V. Churchill, Fourier Series and Boundary Value Problems (McGraw-Hill, New York, 1978), p. 65.

[11] F. Caruso e V. Oguri, Física Moderna - Origens Clássicas e Fundamentos Quânticos (Elsevier, São Paulo, 2006), p. 254.

[12] K.R. Symon, Mecânica (Editora Campus, Rio de Janeiro, 1982), p. 88.

[13] G.B. Arfken and H.J. Weber, Mathematical Methods for Physicists (Academic Press, New York, 1995), p. 137. 